\title{
POLARIZATION OF IO-CM RADIATION OF THE CRAB NEBULA
}

\author{
A. D. KUZ'MIN AND V. A. UDAL'TSOV \\ Lebedev Physical Institute, Moscow, U.S.S.R.
}

In October and November 1957 at the Physical Institute of the Academy of Sciences of the U.S.S.R. a search was conducted to detect the plane polarization of the radiation from the $\mathrm{Crab}$ nebula at $9.6-\mathrm{cm}$ wavelength [1].

The observations were carried out with the $31-\mathrm{m}$ radiotelescope of the Crimean radio-astronomical station [2], which had an immovable parabolic reflector placed in the meridian plane on the declination of the Crab nebula. The conical horn was used as a feed. The polarization-modulation method [3] was used. The polarization modulator (Fig. 1) was a circular waveguide with a ferrite connected to a rectangular waveguide that served as an analyzer. The ferrite rotator $\phi$, placed on the axis of the circular waveguide, was in the longitudinal magnetic field of the solenoid $C$. This field varied according to a cosine law with a modulation frequency equal to $72 \mathrm{c} / \mathrm{s}$. The rotation of the wave's polarization plane was caused by the Faraday effect. A rotation angle equal to \pm 45 degrees was chosen. This accomplished alternate reception and comparison of signals in two perpendicular polarization planes (directions $x$ and $y$ on Fig. 1). By putting an absorption plate into the circular waveguide ahead of the ferrite, the polarization modulator was converted into an amplitude one for receiving the total flux of the Crab nebula. The total flux component in the direction perpendicular to the absorption plate was compared with the thermal radiation of the plate by the amplitude modulator. An S-band receiver was used with the following parameters: noise factor, 10; bandwidth of intermediate frequency amplifier, $5 \mathrm{Mc} / \mathrm{s}$; time constant, 20 seconds. The radiometer's sensitivity was equal to 0.6 to $0.9^{\circ} \mathrm{K}$. A 3120 $\mathrm{Mc} / \mathrm{s}$ local oscillator frequency and a $30 \mathrm{Mc} / \mathrm{s}$ intermediate frequency were chosen to ensure signal reception in two channels placed near frequencies equal to $3090 \mathrm{Mc} / \mathrm{s}$ and $3150 \mathrm{Mc} / \mathrm{s}$, respectively. The bandwidth of each channel was equal to $5 \mathrm{Mc} / \mathrm{s}$. The registration of the signal was carried out by a potentiometer-type recorder элл-09.

The measurement of the received radiation intensity was carried out by the absorption plate, which was put into the circular waveguide in the direction of $x$ (Fig. 1) ahead of the ferrite modulator. The radiation difference between the plate and the sky produced the calibrating step on the recorder.

The series of observations was carried out with different orientations of the analyzer in order to detect the polarization and measure its value and direction. 


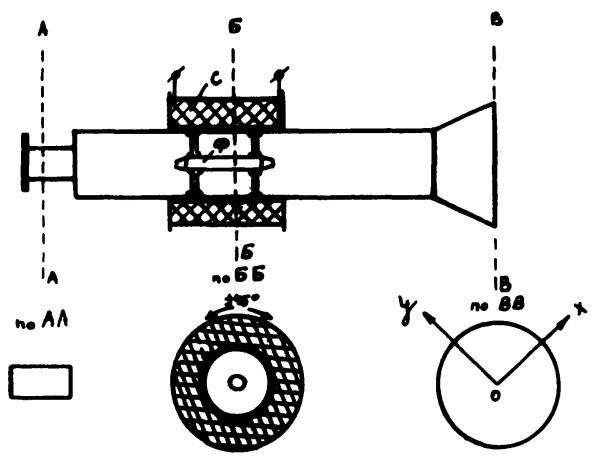

FIg. 1. The circuit of the ferrite-polarized modulator.

In each position of the analyzer two or more measurements were made to increase the reliability of the results. One of the records of this series, measured at position angle of the analyzer equal to $142^{\circ}$, is shown in Fig. 2; also the record of one of the components of total radiation and the calibration step is shown. With a voltage divider in the low-frequency amplifier, the value of this step measured $90^{\circ} \mathrm{K}$.

The value of the useful signal in the direction of the preferred polarization was about 6 degrees. Thus, the signal exceeded the fluctuation by a factor of 7 to 10 . The observations were carried out at night, therefore the interference level was low and hardly decreased the sensitivity of the equipment.

To avoid possible mistakes we made a careful analysis of the parasitic factors that might polarize unpolarized radiation. Our aerial system, having axial symmetry, has the following factors: a difference of antenna radiation patterns in the $E$ and $H$ planes, a cross-polarization, an elongation of the radiation source, and a possible anisotropy of the reflector.

Fig. 3 shows graphically the results of all polarization measurements, in which the antenna temperature $T_{a}$ of the polarized signal is plotted against the position angle of the analyzer. It shows the variation between the value and sign of the polarized signal according to the cosine of twice the position angle. A cosine curve that has the best approximation to the experimental points is shown in the same figure.

The type of polarization, examined by means of a quarter-wave plate, was shown to be linear. The direction of the polarization is defined by a position angle equal to $142 \pm 5$ degrees and coincides with the elongation direction of the Crab nebula. The value of the polarization averaged over the whole nebula is equal to $3 \pm 0.5$ per cent of the total flux.

The polarized radiation in the optical and radio bands of the Crab nebula confirms the assumption advanced by Shklovskii [4] of a common synchrotron mechanism of electron radiation in a magnetic field for optical and radio emission, and shows the presence in the nebula of an electron component of the primary cosmic rays with energy equal to $10^{7}$ to $10^{12} \mathrm{eV}$.

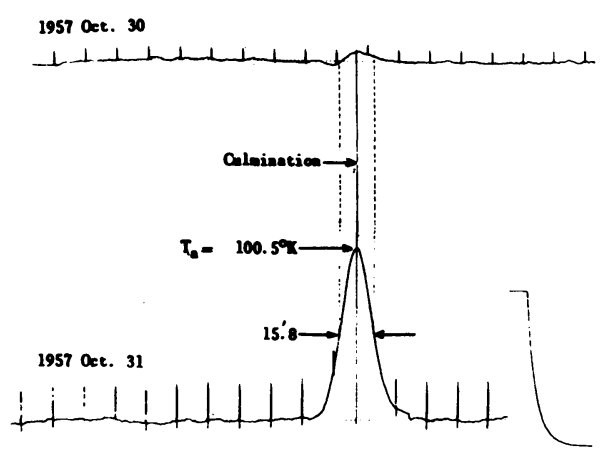

Fig. 2. Samples of the records: $(a)$ polarized component 1957 October $30, \phi=142^{\circ}$, $\Delta T=0.62^{\circ} \mathrm{K} ;(b)$ unpolarized component and calibrating step equal to $90^{\circ} \mathrm{K}, \Delta T$ $=0.78^{\circ} \mathrm{K}$. 


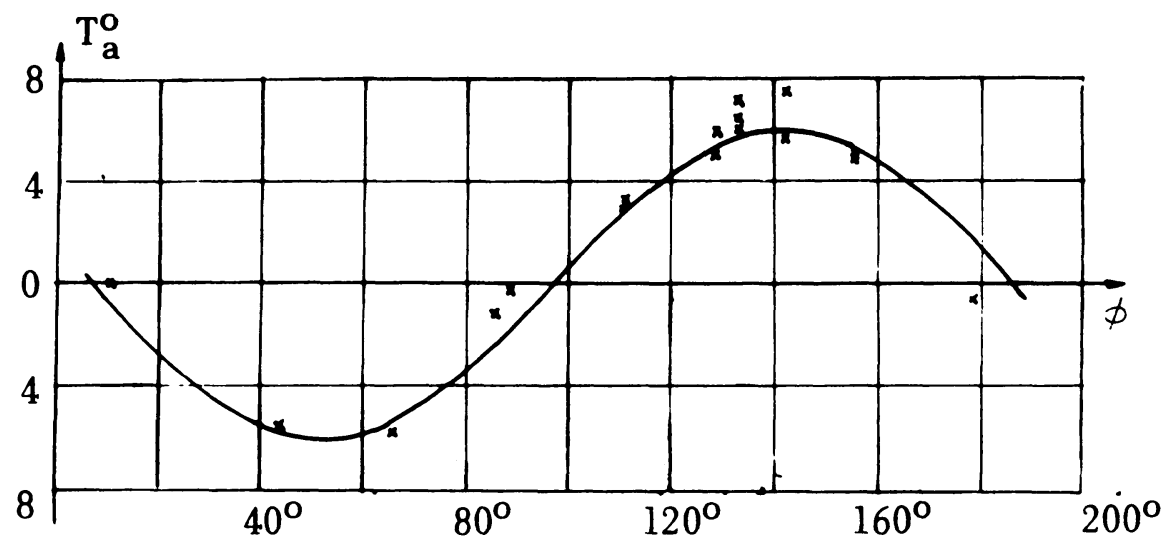

FIG. 3. The dependence of antenna temperature $T_{a}$ of polarized signal on the position angle $\phi$ of analyzer : $x=$ the experimental points; $-=$ the approximation curve $T_{a}$ $=6^{\circ} \mathrm{K}\left(\phi=142^{\circ}\right)$.

The degree of polarization observed by us is less than in the optical range [5] and at the $3.15 \cdot \mathrm{cm}$ wavelength [6]. It may be caused by Faraday dispersion in interstellar space, or in the nebula itself. Taking the following parameters of interstellar gas as the most probable in the plane of the galactic equator $H=3 \times 10^{-6}$ gauss, $N_{e}=0.1 \mathrm{~cm}^{-3}$ and $l=3 \times 10^{19} \mathrm{~cm}$, we have in our case a depolarizing angle $\Delta \psi=2 \times 10^{6} \omega^{-3} N_{e} H l \Delta \omega \cos \alpha=0.05^{\circ}$ for the magnetic cloud. In the above calculation $\Delta \omega=2 \pi \times 2\left(f_{i j}+\frac{1}{2} \Delta f_{i \jmath}\right)=$ $2 \pi \times 65 \times 10^{6}$, because signal reception was carried out by direct and image channels. The size of magnetic cloud is about 10 parsecs. The distance to the Crab nebula is about 1100 parsecs. Therefore, the number of magnetic clouds in the line of sight is 100 and the total depolarization angle is not more than 5 degrees. Consequently, the depolarization effect of interstellar gas is very small, and we must look for its cause in the Crab nebula itself.

Furthermore, it is necessary to remember that a considerable part of the radiation comes from the peripheral area of the nebula, where the magnetic fields are less homogeneous.

The authors wish to express their thanks to V. V. Vitkevich for his having suggested the present investigation and for his constant interest during its execution. They are also grateful to their colleagues of the Physical Institute for many fruitful discussions.

\section{REFERENCES}

[1] Kuz'min, A. D. and Udal'tsov, V. A. Astr. Circ. Acad. Sci. U.S.S.R. No. 187, p. 14, 1957.

[2] Vitkevich, V.V. and Udal'tsov, V. A. Radio Engineering and Electronics, U.S.S.R. 2, 1548, 1957.

[3] Kaidanovskii, N. L., Mirzabekian, E. G., and Khaikin, S. E. Transactions of the 
Fifth Conference on Cosmogonical Questions. Moscow (Acad. Sci. U.S.S.R. Press), 1956, pp. 113-21.

[4] Shklovskii, I. S. Dokl. Akad. Nauk S.S.S.R. 90, 1983, 1953.

[5] Oort, J. H. and Walraven, T. H. B.A.N. 12, 285, 1956.

[6] Mayer, C. H., McCullough, T. P., and Sloanaker, R. M. Ap. J. 126, 468, 1951.

\section{Discussion}

Cohen: Was the radiation examined for circular polarization?

Vitkevich: Yes; but it was not found.

Gold: At what bandwidth was this $10-\mathrm{cm}$ polarization found?

Vitkevich: Energy was received simultaneously in two $5-\mathrm{Mc} / \mathrm{s}$ channels separated by $65 \mathrm{Mc} / \mathrm{s}$.

Shakeshaft: In view of the possible rotation of the plane of polarization due to the Faraday effect in interstellar space, is it to be expected that the agreement in position angle between the observed planes of polarization at 3 and $10 \mathrm{~cm}$ should be so good?

Vitkevich: The Faraday rotation angle is not large.

Evans: From my own observations I would expect that at $10 \mathrm{~cm}$ errors as large as 5 degrees could be produced by the Faraday effect in the earth's ionosphere.

Shakeshaft: Has Professor Oort made any estimate of the rotation angle of the plane of polarization to be expected at $10-\mathrm{cm}$ wavelength?

Oort: I don't recall what rotation we estimated for $3 \mathrm{~cm}$. I think it was about 5 or 10 degrees. It is somewhat surprising to find that the position angle at $10 \mathrm{~cm}$ coincides so well with the optical one, particularly when the polarization is only one-half of that observed by Mayer at $3 \mathrm{~cm}$.

Scheuer: How does the percentage polarization found on $10 \mathrm{~cm}$ compare with the percentage found on $3 \mathrm{~cm}$ ?

Oort: Seven per cent at $3 \mathrm{~cm}$, as measured by Mayer. 